\title{
Pneumomediastino espontâneo (síndrome de Hamman) relato de dois casos
}

\author{
Spontaneous pneumomediastinum (Hamman's syndrome) two cases report
}

\begin{abstract}
Amanda Bishop Perseguim ${ }^{1}$, Douglas Alexandre Rizzanti Pereira², Luciano Bertipaglia Fiori ${ }^{3}$, Marcelo Manzano Said ${ }^{4}$, Marcus Vinícius Roncada Peres 5 , José Luis Braga de Aquino ${ }^{6}$
\end{abstract}

Perseguim AB, Pereira DAR, Fiori LB, Said MM, Peres MVR, Aquino JLB. Pneumomediastino espontâneo (síndrome de Hamman) relato de dois casos / Spontaneous pneumomediastinum (Hamman's syndrome) two cases report. Rev Med (São Paulo). 2016 jul.set.;95(3):138-41.

\begin{abstract}
RESUMO: Objetivo: Descrever dois casos de pneumomediastino espontâneo na mesma unidade de serviço, em pacientes jovens de sexo diferentes e que apresentaram boa evolução com tratamento conservador. Métodos: O primeiro caso é de um paciente do sexo masculino, 18 anos, com queixa de cervicalgia à direita e disfonia há 2 dias. Negou traumatismos ou ingesta de corpos estranhos, esforços físicos intensos, acessos de tosse e vômitos ou uso de drogas. TC de tórax e pescoço revelou presença de enfisema subcutâneo cervical anterior bilateral, presença de pneumomediastino e ausência de pneumotórax. O segundo caso a ser analisado trata-se de uma paciente do sexo feminino, 16 anos, com queixa de dispneia súbita e mudança do padrão de voz (nasalada). Identificou-se crepitação fina à palpação cervical sugestiva de enfisema subcutâneo. Negou qualquer causa provável de pneumomedisatino secundário. TC de tórax e pescoço revelou pneumomediastino acentuado com extensão para os espaços cervicais profundos. Resultado: Ambos os casos partilham de sinais e sintomas previamente descritos
\end{abstract}

na literatura e tiveram seus diagnósticos obtidos por meio de tomografia computadorizada de tórax. O tratamento sintomático associado a repouso foi eficaz em ambas situações. Conclusões: Pneumomediastino espontâneo pode ser subdiagnosticado devido aos seus sinais e sintomas inespecíficos, contribuindo para seu caráter raro. É importante estabelecer-se os diagnósticos diferenciais e eventos a que os pacientes estiveram expostos. Uma anamnese detalhada acompanhada de aparatos radiológicos permitem o diagnóstico preciso e tratamento correto.

Descritores: Enfisema mediastínico; Enfisema subcutâneo; Fibrose pulmonar.

ABSTRACT: Objective: Describe two cases of spontaneous pneumomediastinum in the same service unit in young patients of different sex and that showed good progress with conservative treatment. Methods: The first case is a male patient, 18, with neck pain on the right side complaints and dysphonia for 2

Fonte de financiamento: nenhuma.

Trabalho realizado pela Disciplina de Cirurgia do Tórax da FM/CC, PUC-Campinas/HMCP 2015.

E-Pôster - categoria Clínica Cirúrgica, XXXI Congresso Brasileiro de Cirurgia, Curitiba, PR, 2015. Pôster - categoria Clínica Cirúrgica, XIII Pré-Congresso Médico Acadêmico Samuel Pessoa, inscrito sob no 114279 na CNA, Campinas, SP, 2015. Pôster - Clínica Cirúrgica, X Simpósio Paulista das Ligas do Trauma, 2015, Escola Paulista de Medicina. Premiado no X Simpósio Paulista das Ligas do Trauma.

1. Acadêmica do $5^{\circ}$ ano da FCM da PUC-Campinas, autora responsável por apresentações e publicação. E-mail: amandaperseguim@ hotmail.com.

2. Cirurgião de Cabeça e Pescoço assistente da PUC-Campinas. E-mail: med.dpereira@gmail.com.

3. Residente do $2^{\circ}$ ano de Cirurgia Geral da PUC-Campinas. E-mail: fiorilb@gmail.com.

4. Médico assistente do Serviço de Cirurgia Torácica da PUC-Campinas. E-mail: gmsaid@uol.com.br.

5. Médico assistente do serviço de cirurgia geral da PUC-Campinas. E-mail: dom pedro xxv@yahoo.com.br.

6. Médico assistente e Chefe do Serviço de Cirurgia Torácica e Assistente do Serviço de Cirurgia de Cabeça Pescoço da PUC-Campinas. E-mail: jlaquino@sigmanet.com.br.

Endereço para correspondência: Amanda Bishop Perseguim. Av. Antônio Carvalho de Miranda, 720, Jardim Miranda, Residencial das Colinas, apto 172 MA. Campinas, SP. E-mail: amandaperseguim@hotmail.com. 
days. Denied injuries or ingestion of strange bodies, heavy physical exertion, coughing and vomiting or drug use. CT of the chest and neck revealed the presence of bilateral anterior cervical subcutaneous emphysema, pneumomediastinum presence and absence of pneumothorax. The second case to be analyzed it is a female patient, 16 years old, complaining of sudden dyspnea and standard voice change (nasal). It was identified to fine crackling suggestive cervical palpation of subcutaneous emphysema. He denied any probable cause of secondary pneumomedistino. CT of the chest and neck revealed sharp pneumomediastinum extending to the deep neck spaces. Result: Both cases share of signs and symptoms previously

\section{INTRODUÇÃO}

$\mathrm{P}$ neumediastino espontâneo é uma condição rara, de caráter benigno e evolução autolimitada ${ }^{1}$, definida como presença de ar livre no mediastino sem nenhum fator desencadeante aparente ${ }^{2}$. Foi originalmente descrito em 1939 por Louis Hamman ${ }^{3}$. A crepitação precordial durante a sístole na ausculta cardíaca é considerada patognomônico da síndrome embora tenha baixa frequência ( $50 \%$ dos casos).

A fisiopatologia do Pneumomediastino Espontâneo foi esclarecida em 1944 por Macklin ${ }^{4}$. Consiste no aumento súbito de pressão intra-alveolar, levando ao surgimento de um gradiente de pressão e consequente ruptura de alvéolos pulmonares. Dessa maneira ocorre a dissecção do ar pelo hilo através de fáscias peribroncovasculares, pela fáscia mediastinal, podendo atingir os tecidos subcutâneos do tórax, membros superiores, pescoço e retroperitôneo ${ }^{1}$.

Devido a sinais e sintomas inespecíficos essa condição pode ser subdiagnosticada contribuindo para sua baixa incidência. Além disso, os sintomas não são muito específicos, podendo ter sinais despercebidos e difíceis de identificar radiologicamente ${ }^{2}$. O pneumomediastino espontâneo responde por $1 \%$ dos casos totais de pneumomediastino ${ }^{5}$. É mais visto em pacientes longilíneos e do sexo masculino ${ }^{1}$ entre 17 e 25 anos, sendo a proporção do sexo masculino e do sexo feminino de $8 / 1^{6}$.

As principais causas de PE envolvem eventos que expõem o paciente a mudanças bruscas de pressão intratorácica como exercícios físicos, trabalho de parto, mergulhos em grandes profundidades, vômitos, acessos de tosse, cetoacidose diabética, inalação de drogas, tabagismo, exacerbações de asma. Entretanto, em 30\% dos casos não é possível identificar o fator desencadeante ${ }^{1}$.

Dor retroesternal com piora à inspiração profunda e a mudança de decúbito, dispneia e enfisema subcutâneo são as manifestações clínicas mais comuns ${ }^{2}$. O paciente pode ainda apresentar tosse, pneumotórax, disfonia, disfagia e dor cervical. Enfisema subcutâneo é descrito como sinal de boa evolução clinica, pois, o extravasamento aéreo para o tecido subcutâneo preveniria o aumento da pressão intramediastinal que poderia comprimir os grandes vasos ${ }^{7}$. described in the literature and had their diagnoses obtained by computed tomography. Symptomatic treatment associated with home was effective in both situations. Conclusions: Spontaneous pneumomediastinum may be underdiagnosed because of its nonspecific signs and symptoms, contributing to their unique character. It is important to establish the differential diagnoses and events to which patients were exposed. Accompanied a detailed history of radiological devices allow precise diagnosis and correct treatment.

Keywords: Mediastinal emphysema; Subcutaneous emphysema; Pulmonary fibrosis.

O diagnóstico pode ser obtido através de exames de imagem, radiografia simples do tórax e a tomografia computadorizada de tórax, sendo esta o padrão ouro para o diagnóstico. Os exames laboratoriais podem se mostrar inespecíficos, mostrando discreto aumento de Proteína $\mathrm{C}$ reativa e leucocitose moderada ${ }^{7,8}$.

Entre os diagnósticos diferenciais há: perfuração esofágica (Síndrome de Boerhaave), pericardite, ruptura de árvore traqueobrônquica e pneumotórax hipertensivo ${ }^{1}$.

O tratamento é sintomático e geralmente consiste de analgesia, oxigenoterapia e repouso. Não há consenso sobre o uso de antibióticos ${ }^{1}$, podendo o mesmo pode ser prescrito sob justificativa da prevenção de mediastinite ${ }^{7}$. Seu uso pode ser questionado considerando a possibilidade de se mascarar lesões ocultas em caso de ruptura de esôfago ${ }^{9}$.

\section{RELATO DE CASOS}

O Primeiro caso refere-se a paciente do sexo masculino, de 18 anos, que relata no serviço de urgência cervicalgia a direita com 2 dias de evolução, acompanhada de disfonia. Negava traumatismos, procedimentos invasivos, acessos de tosse, vômito, intenso esforço físico ou uso de drogas inaladas e medicações. Negava ainda dor torácica, dispneia e disfagia. Ao exame físico: bom estado geral, eupneico, expansilibidade e elasticidade torácica preservadas, murmúrio vesicular diminuído à esquerda, com presença de sibilos difusos. Não apresentava alterações na propedêutica cardíaca e abdominal. Palpação cervical à direita dolorosa, com sinais flogísticos e enfisema subcutâneo. Realizou tomografia computadorizada de tórax e pescoço que revelou enfisema subcutâneo cervical, pneumomediastino e ausência de pneumotórax, conforme Figura 1. Optado por tratamento clinico conservador e antibiótico terapia com administração de amoxicilina clavulanato $3 \mathrm{~g} /$ dia, IV, hidrocortisona $100 \mathrm{mg}$ IV 8/8h, analgésicos e soro de hidratação, associado a repouso e fisioterapia respiratória/ inaloterapia. No quinto dia de internação apresentava ausência do enfisema subcutâneo cervical, diminuição do desconforto respiratório, exames laboratoriais e ecocardiograma inalterados, recebendo então alta médica e seguimento ambulatorial. 


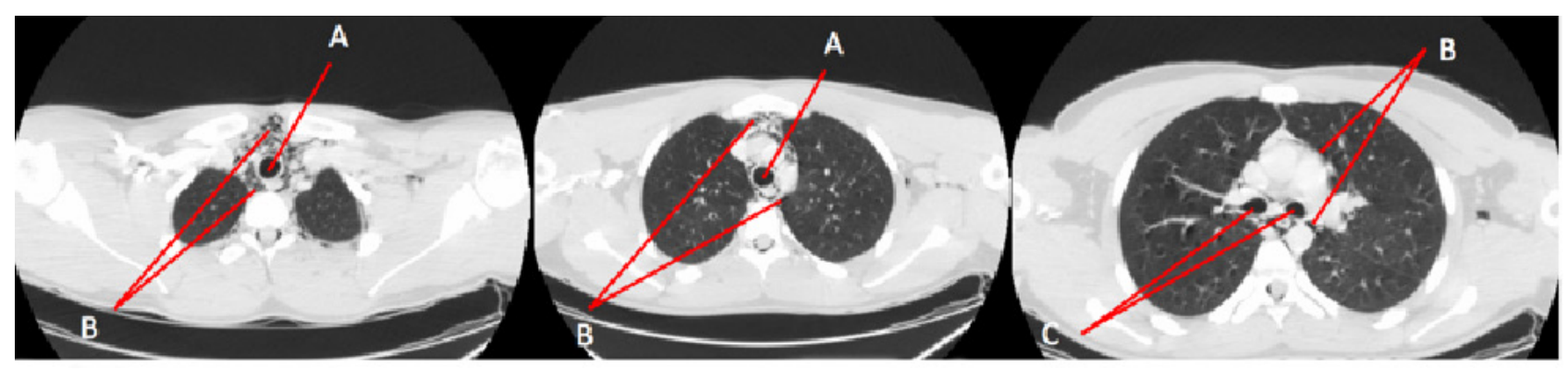

Figura 1. Caso 1. Seta A - hipodensidade compatível com ar, visualizada na traquéia do paciente. Seta B - hipodensidade mediastinal, semelhante a A,porém fora da topografia da traquéia e brônquios. Seta $\mathrm{C}$ - hipodensidade compatível com ar em topografia de brônquiofontes direito e esquerdo

O segundo caso trata-se de paciente do sexo feminino, de 16 anos, que deu entrada no serviço de urgência relatando dispnéia súbita de grande intensidade, ao repouso, e mudança no padrão da voz - que passou a ser anasalada 1 dia antes do atendimento. Paciente também negou fatores predisponentes já mencionados. Ao exame físico, a paciente apresentava bom estado geral, sem sinais de insuficiência respiratória, murmúrio vesicular diminuído globalmente, sem ruídos adventícios.
Não apresentava alterações na propedêutica cardíaca e abdominal. Presença de enfisema subcutâneo. A tomografia computadorizada que revelou pneumomediastino, com extensão para os espaços cervicais profundos, conforme Figuras 2 e 3 . A conduta clínica foi similar à descrita no caso anterior: repouso, suporte ventilatório, analgesia e antibioticoterapia com Amoxilina clavulanato 3g/dia, IV. Paciente evoluiu bem, com resolução das queixas, obtendo alta médica após 2 dias de internação.

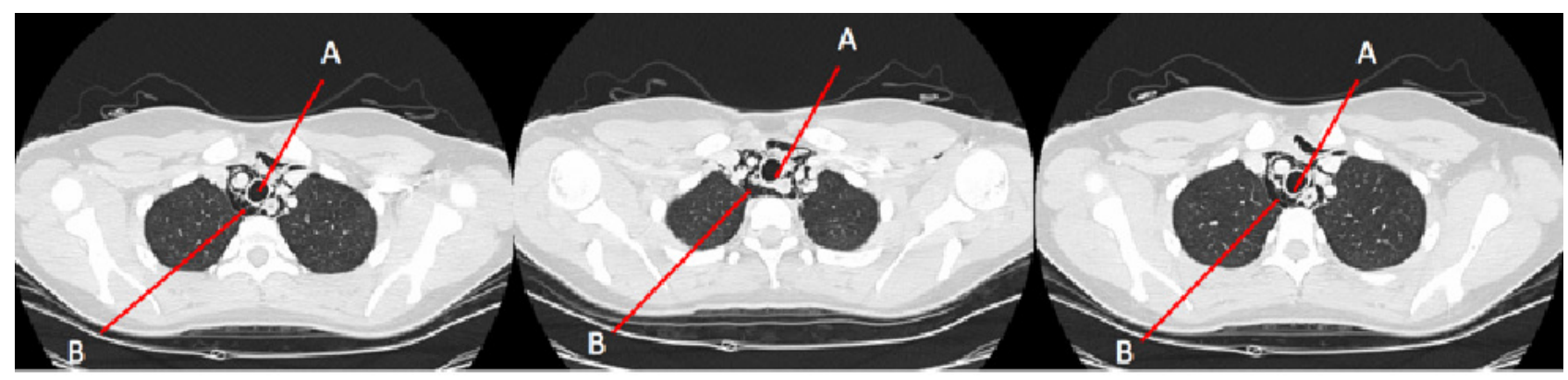

Figura 2. Caso 2 - Seta A - Hipodensidade compatível coma r, visualizada na traqueia do paciente. Seta B - Hipodensidae mediastinal, semelhante a A, porém fora da topografia da traqueia e brônquios

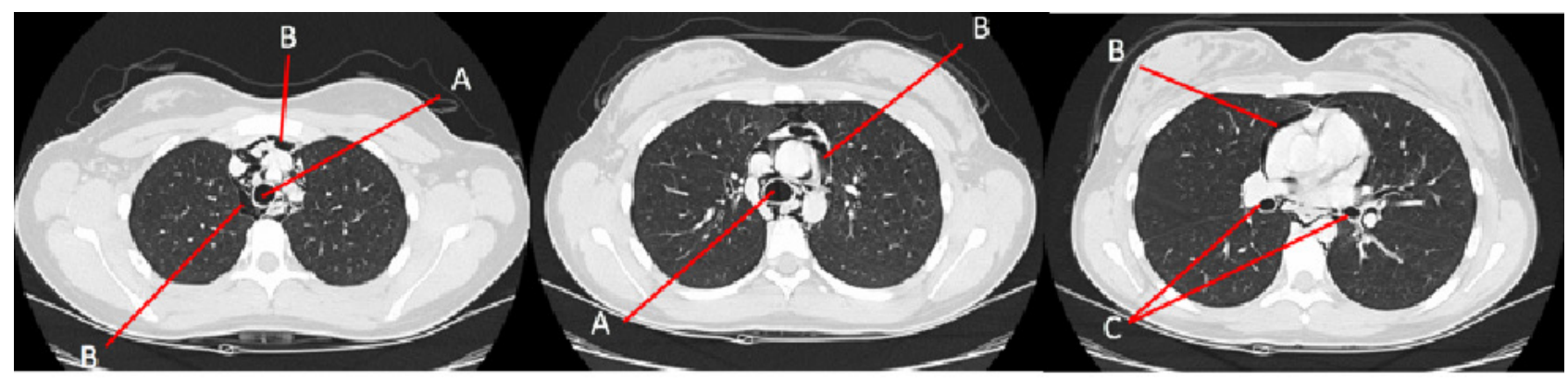

Figura 3. Caso 2 - Seta A - Hipodensidade compatível com ar, visualizada na traqueia do paciente. Seta B - Hipodensidade mediastinal, semelhante a A, porém fora da Topografia da traqueia e brônquios. Seta C - Hipodensidade compatível com ar em topografia de brônquio-fontes direito esquerdo

\section{DISCUSSÃO}

Pneumomediastino espontâneo é uma condição rara, de caráter benigno e evolução autolimitada', usualmente sem nenhum fator desencadeante aparente ${ }^{2}$, dor retroesternal com piora à inspiração profunda e mudança de posição, dispnéia e enfisema subcutâneo são as manifestações clínicas mais comuns.

Ambos os casos partilham de apresentações clinicas encontradas na literatura como dispnéia, enfisema subcutâneo e disfonia. O diagnóstico foi confirmado através de tomografia computadorizada de tórax, havendo 
penumomediastino e dissecção para região cervical com ausência de pneumotórax. O tratamento clínico conservador se valendo de sintomáticos e repouso foi eficaz nos dois casos acima descritos indo de encontro com a evolução benigna descrita na literatura. Foi ainda optado por antibioticoprofilaxia nos dois casos.

\section{REFERÊNCIAS}

1. Leme M, Martinez M, Monteiro C, Lotufo J. Pneumomediastino Espontâneo em adolescente após visita a parque de diversões - relato de caso. Rev Pediatria (São Paulo). 2010;32(4):298-300.

2. Macia I, Moya J, Ramor R, Morera R, Escobar I, Saumench J, Perna V, Rivas F. Spontaneous Pneumomediastinum: 41 cases. Eur J CardioThoracic Surg. 2007;31(6):1110-14. doi: 10.1016/j. ejcts.2007.03.00.

3. Hamman L. Spontaneous Mediastinal Emphysema. Bull Johns Hopkins Hosp. 1939;64:1-21.

4. Caceres M, Ali S, Braud R, Wieman D, Garret HE. Sponteaneous pneumomediastinum: a comparative study and review of the literature. Ann Thorac Surg. 2008;86:962-6. doi: http://dx.doi.org/10.1016/j. athoracsur.2008.04.067.

5. Ruiz-Ruiz FJ, Sampériz A, Rubio T, Escolar F. Neumomediastino espontáneo. Anales Sis San Navarra. 2006;29(2):275-8. Disponible en: http://scielo.isciii.es/scielo.php?script $=$ sci arttext\&pid=S1137-66272006003300111\&lng=es.

6. Semedo FHMA, Silva RS, Pereira S, Alfaiate T,

\section{CONCLUSÕES}

Pneumomediastino espontâneo, apesar de condição rara deve ser considerado como diferencial em paciente jovem com dor torácica, enfisema subcutâneo cervical ou alteração de voz. A tomografia computadorizada permite o diagnóstico preciso e o tratamento clínico conservador constitui o tratamento de eleição.

Costa T, Fernandez P, et al. Pneumomediastino espontâneo: relato de um caso. Rev Assoc Med Bras. 2012;58(3):355-7. doi: 10.1590/S010442302012000300017.

7. Takada K, Matsumoto S, Hiramatsu T, Kojima E, Watanabe H, Sizu M, Okachi S, Ninomiya K. Management of spontaneous pneumomediastinum based on clinical experience of 25 cases. Division of Respiratory and Allergy Medicine, Komaki Municipal Hospital, 1-20 Johbuji, Komaki 485-8520, Japan. Respir Med. 2008;102(9):1329-34. doi: 10.1016 / j.rmed.2008.03.023.

8. Silva FA, Barros CD, Raddatz EA. Neumomediastino espontáneo (síndrome de Hamman), una entidad poco frecuente no siempre reconocida. Rev Chil Cir. 2013;65(5):442-7. doi: $10.4067 / \mathrm{S} 0718$ 40262013000500013.

9. Al-Mufarrej F, Badar J, Gharagozloo F, Tempesta B, Strother E, Margolis M. Spontaneous Pneumomediastinum: diagnostic and therapeutic interventions. J Cardiothorac Surg. 2008;3:59. doi 10.1186/1749-80-90-3-59. 